TRANSACTIONS OF THE

AMERICAN MATHEMATICAL SOCIETY

Volume 358, Number 9, September 2006, Pages 4045-4055

S 0002-9947(05)03798-0

Article electronically published on September 22, 2005

\title{
SEIFERT-FIBERED SURGERIES WHICH DO NOT ARISE FROM PRIMITIVE/SEIFERT-FIBERED CONSTRUCTIONS
}

\author{
THOMAS MATTMAN, KATURA MIYAZAKI, AND KIMIHIKO MOTEGI
}

Dedicated to Cameron McA. Gordon on the occasion of his 60th birthday

\begin{abstract}
We construct two infinite families of knots each of which admits a Seifert fibered surgery with none of these surgeries coming from Dean's primitive/Seifert-fibered construction. This disproves a conjecture that all Seifert-fibered surgeries arise from Dean's primitive/Seifert-fibered construction. The $(-3,3,5)$-pretzel knot belongs to both of the infinite families.
\end{abstract}

\section{INTRODUCTION}

Let $K$ be a knot in the 3 -sphere $S^{3}$. Then we denote by $(K ; \gamma)$ the 3 -manifold obtained by $\gamma$-surgery on $K$, i.e., by attaching a solid torus to $S^{3}-\operatorname{int} N(K)$ in such a way that $\gamma$ bounds a meridian disk of the filling solid torus. Using the preferred meridian-longitude pair of $K \subset S^{3}$, we parametrize slopes $\gamma$ of $K$ by $r \in \mathbb{Q} \cup\{\infty\}$; then we also write $(K ; r)$ for $(K ; \gamma)$.

We begin by recalling Berge's [1] construction, an explicit construction which yields several infinite families of knots each admitting a lens space Dehn surgery.

Let $K$ be a knot contained in a genus two Heegaard surface $F$ for $S^{3}$, i.e., $S^{3}=H \cup_{F} H^{\prime}$, where $H$ and $H^{\prime}$ denote genus two handlebodies. Suppose that $K$ is nontrivial and that the manifolds $H(K)$ and $H^{\prime}(K)$ are both solid tori, where $H(K)$ (resp. $H^{\prime}(K)$ ) is obtained by attaching a 2-handle to $H$ (resp. $H^{\prime}$ ) along $K$. The isotopy class in $\partial N(K)$ of the curve(s) in $\partial N(K) \cap F$ is called the surface slope of $K$ with respect to $F$. Then by performing Dehn surgery on $K$ along the surface slope $\gamma$, we obtain a 3 -manifold $(K ; \gamma)=H(K) \cup H^{\prime}(K)$, which is a lens space. It cannot be $S^{2} \times S^{1}$ by [11, nor $S^{3}$ by [14]. This construction is called Berge's construction or the primitive/primitive construction and such a knot $K$ is said to be primitive/primitive with respect to $F$.

In [1] Berge suggested the following. See also [13].

Conjecture 1.1. If $(K ; \gamma)$ is a lens space, then this surgery arises from Berge's construction.

Received by the editors January 20, 2003 and, in revised form, June 28, 2004.

2000 Mathematics Subject Classification. Primary 57M25.

Key words and phrases. Dehn surgery, hyperbolic knot, Seifert fiber space, primitive/Seifertfibered construction.

The first author was supported in part by grants from NSERC and FCAR.

The second author was supported in part by Grant-in-Aid for Scientific Research (No. 40219978), The Ministry of Education, Culture, Sports, Science and Technology, Japan.

(C)2005 American Mathematical Society Reverts to public domain 28 years from publication 
Dean [7, 8] made a natural modification to Berge's construction; suppose that $K$ is as before except that $H^{\prime}(K)$ is now a Seifert fiber space over the disk with two exceptional fibers. Then for the surface slope $\gamma,(K ; \gamma)$ is a Seifert fiber space over $S^{2}$ with at most three exceptional fibers or a connected sum of two lens spaces. If $K$ is hyperbolic, then the cabling conjecture [12] states that the latter cannot occur. This construction is called Dean's construction or the primitive/Seifertfibered construction and such a knot $K$ is said to be primitive/Seifert-fibered with respect to $F$.

The notion of primitive/Seifert-fibered construction has been slightly generalized by allowing the possibility that $H^{\prime}(K)$ is a Seifert fiber space over the Möbius band with one exceptional fiber [10, [18. In the following, we use the term primitive/Seifert-fibered construction (or knot) in this generalized sense.

In analogy with Conjecture 1.1, Dean [7] and Gordon [13] asked:

Question 1.2. If $(K ; \gamma)$ is a Seifert fiber space other than a lens space, then does this surgery arise from a primitive/Seifert-fibered construction?

Many examples of Seifert-fibered surgeries (see, for example, 4, [5, 9] and [10]) have been constructed using the Montesinos trick ([19], 3]). Recently, in [10, Eudave-Muñoz has shown that all known examples of Seifert fibered surgeries constructed by the Montesinos trick can be explained by Dean's construction. Furthermore, Seifert fibered surgeries on twisted torus knots in [17 can also be explained by such constructions [18.

On the other hand, in the present note we demonstrate the following which answers the question above in the negative. A knot $K$ is strongly invertible if there is an orientation preserving involution of $S^{3}$ which leaves $K$ invariant and reverses an orientation of $K$; primitive/Seifert-fibered knots are shown to be strongly invertible.

Theorem 1.3. There is an infinite family of non-strongly invertible knots each of which admits a Seifert-fibered surgery with none of these surgeries arising from the primitive/Seifert-fibered construction. For example, the $(-3,3,5)$-pretzel knot belongs to the family.

Very recently Hyung-Jong Song has observed that the 1-surgery of the $(-3,3,3)$ pretzel knot is a Seifert-fibered surgery, but does not arise from the primitive/Seifertfibered construction. In contrast with our examples, the $(-3,3,3)$-pretzel knot is strongly invertible; but it has cyclic period 2 and tunnel number greater than one like ours.

In his thesis [15, the first author observed that the $(-3,3,5)$-pretzel knot has a small Seifert-fibered surgery by experiments via Weeks' computer program SnapPea. This observation is the starting point of our study.

\section{EXAMPLES}

We shall say that a Seifert fiber space is of type $S^{2}\left(n_{1}, n_{2}, n_{3}\right)$ if it has a Seifert fibration over $S^{2}$ with three exceptional fibers of indices $n_{1}, n_{2}$ and $n_{3}\left(n_{i} \geq 2\right)$.

Example 1. Let $K \cup t_{1}$ be the two component link of Figure 1.

Here $K$ is the Montesinos knot given by the triple of rational tangles $(1 / 3,-1 / 3,-1 / 5)$, which is often called the $(-3,3,5)$-pretzel knot. (We adopt Bleiler's convention [2] on the parametrization of rational tangles.) Let $K_{n}$ ( $n$ is 


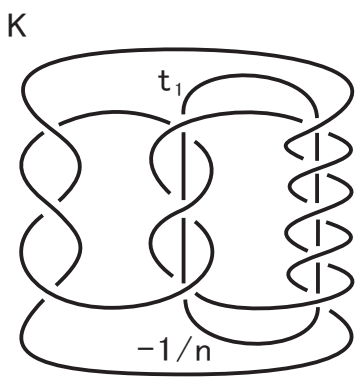

FIGURE 1.

possibly zero) be the knot obtained from $K$ by performing $-1 / n$-surgery on $t_{1}$. Equivalently, $K_{n}$ is obtained by doing $n$-twisting along $t_{1}$. Then $K_{n}$ enjoys the following properties:

(1) $K_{n}$ is a hyperbolic knot,

(2) $K_{n}$ has cyclic period 2, but is not strongly invertible,

(3) the tunnel number of $K_{n}$ is 2 , and

(4) $\left(K_{n} ; 1\right)$ is a Seifert fiber space of type $S^{2}(3,5,|15 n+4|)$.

Before verifying properties (1)-(4) we observe that $\left\{K_{n}\right\}$ is the family of Theorem 1.3

Proof of Theorem 1.3. Properties (2) and (4) show that $K_{n}$ is not strongly invertible and admits a Seifert-fibered surgery. Assume for a contradiction that $K_{n}$ is primitive/Seifert-fibered; then $H\left(K_{n}\right)$ is a solid torus for an unknotted genus 2 handlebody $H$ with $K \subset \partial H$. First we show that $K_{n}$ has tunnel number 1 following [7. By [25], there is a homeomorphism of the genus two handlebody $H$ after which $K_{n}$ appears as in Figure 2, After pushing $K_{n}$ into $H$, take an arc $t$ as in Figure 2 ,

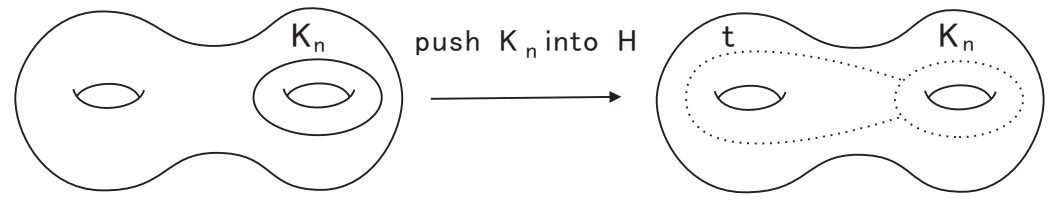

FIGURE 2.

Then $H-\operatorname{int} N\left(K_{n} \cup t\right)$ is the product of a surface and an interval. Thus $S^{3}-$ $\operatorname{int} N\left(K_{n} \cup t\right)=H^{\prime} \cup\left(H-\operatorname{int} N\left(K_{n} \cup t\right)\right)$ is a genus two handlebody, so the knot $K_{n}$ has tunnel number 1 . This then implies that $K_{n}$ is strongly invertible by [21, Lemma 5], a contradiction. Hence the Seifert-fibered surgery does not come from the primitive/Seifert-fibered construction.

$\square$ (Theorem [1.3)

Claim 2.1. $K_{n}$ has cyclic period 2.

Proof. As shown in Figure 3, let $f: S^{3} \rightarrow S^{3}$ be the $\pi$-rotation about $C$ such that $f(K)=K$ and $f\left(t_{1}\right)=t_{1}$. The axis $C$ is disjoint from $K$ and intersects $t_{1}$ in exactly two points. 


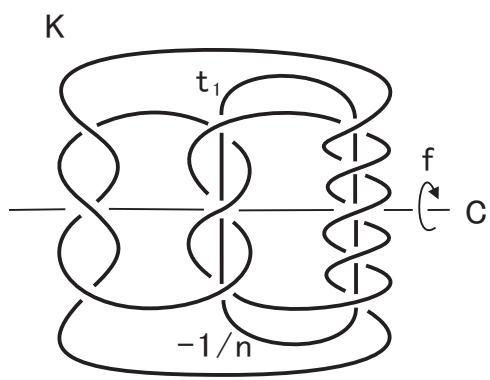

FIGURE 3.

Hence, $f \mid S^{3}-\operatorname{int} N\left(t_{1}\right)$ extends to an involution $\bar{f}$ of $\left(t_{1} ;-1 / n\right) \cong S^{3}$ about an axis $\bar{C}$ such that $\bar{f}\left(K_{n}\right)=K_{n}$ and $K_{n} \cap \bar{C}=\emptyset$. It follows that $K_{n}$ has cyclic period 2 .

$\square$ (Claim 2.1)

Claim 2.2. $\left(K_{n} ; 1\right)$ is a Seifert fiber space of type $S^{2}(3,5,|15 n+4|)$.

Proof. Let $\left(K \cup t_{1} ; 1,-1 / n\right)$ denote the manifold obtained by performing a surgery on the link $K \cup t_{1}$ with surgery slopes 1 for $K$ and $-1 / n$ for $t_{1}$. We will show that $\left(K \cup t_{1} ; 1,-1 / n\right)$ is a Seifert fiber space of type $S^{2}(3,5,|15 n+4|)$.

To prove this we form the quotient by the involution $f: S^{3} \rightarrow S^{3}$ to obtain the factor knot $K_{f}$, the branched knot $c$ which is the image of $C$, and the $\operatorname{arc} \tau_{1}$ which is the image of $t_{1}$ and connects two points in $c$ (Figure 4).

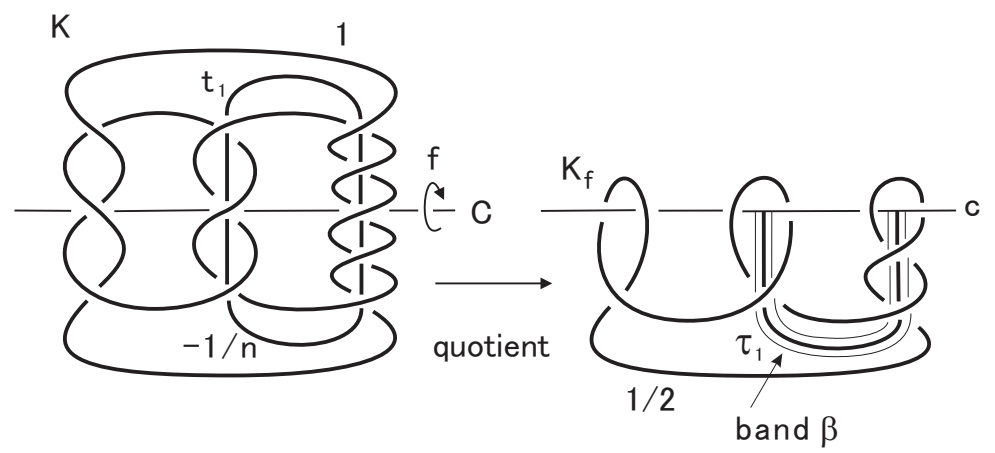

FiguRE 4.

As shown in Figure 4, the factor knot $K_{f}$ is unknotted in $S^{3} / f \cong S^{3}$. Note that 1-surgery on $K$ corresponds to $1 / 2$-surgery on the factor knot $K_{f}$ which is equivalent to (-2)-twisting along $K_{f}$ because $K_{f}$ is unknotted; see Figure 6. We denote the image of $c$ after (-2)-twisting along $K_{f}$ by $c^{\prime}$. Note also that by the Montesinos trick ([19], [3]), $-1 / n$-surgery on $t_{1}$ corresponds to $-1 / n$-untangle surgery (i.e., a replacement of a $1 / 0$-untangle by a $-1 / n$-untangle) on $c^{\prime}$ along $\tau_{1}$ as indicated in Figure 8 . In order to correctly perform the untangle surgery, we keep track of the framing. This can be done by indicating a band $\beta$ whose core is $\tau_{1}$; see Figure 4. (For simplicity, we indicate the band $\beta$ in only two places: just after taking the quotient by the involution $f$, and just before performing the untangle 
surgery.) By an isotopy as in Figures 6 and 7, we see that $c^{\prime}$ is the Montesinos knot given by the triple of rational tangles $(2 / 5,-3 / 4,1 / 3)$. Denote the result of $-1 / n$-untangle surgery on $c^{\prime}$ by $c_{n}^{\prime}$ (Figure 8). Then $c_{n}^{\prime}$ is the Montesinos knot given by the triple of rational tangles $(2 / 5,(11 n+3) /(-15 n-4), 1 / 3)$, and the branched covering space $\left(K \cup t_{1} ; 1,-1 / n\right)$ of $S^{3}$ branched along $c_{n}^{\prime}$ is a Seifert fiber space of type $S^{2}(3,5,|15 n+4|)$. Since the linking number of $K$ and $t_{1}$ is zero, the 1-slope of $K$ corresponds to the 1 -slope of $K_{n}$, and hence $\left(K \cup t_{1} ; 1,-1 / n\right) \cong\left(K_{n} ; 1\right)$. It follows that $\left(K_{n} ; 1\right)$ is a Seifert fiber space of type $S^{2}(3,5,|15 n+4|)$ as required.

$\square$ (Claim 2.2)
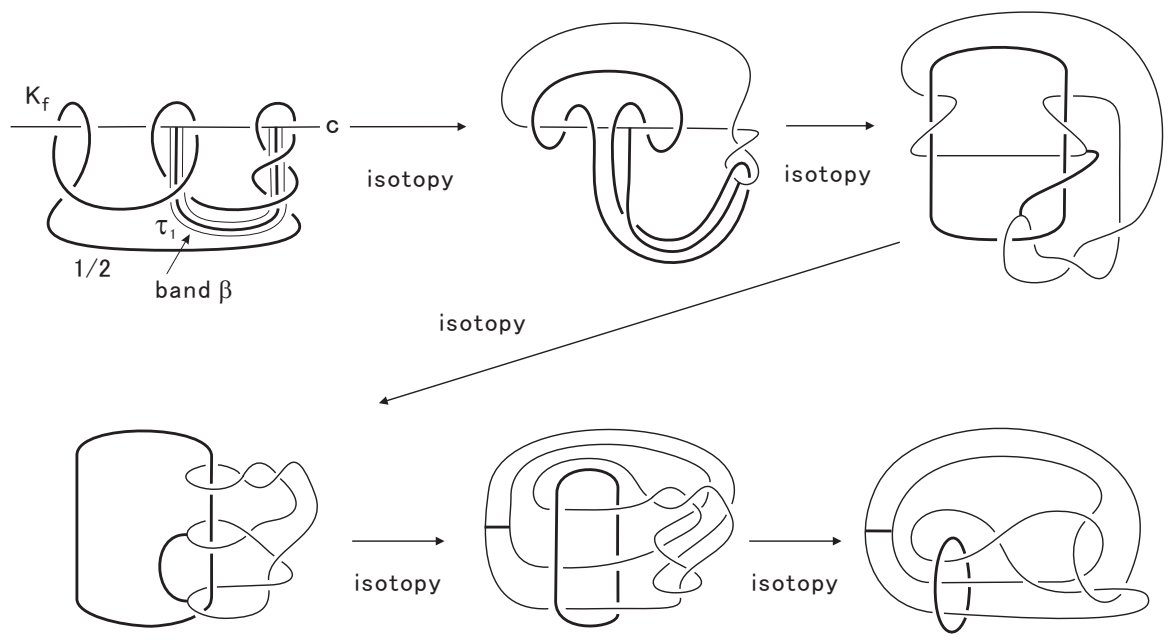

FiguRe 5.
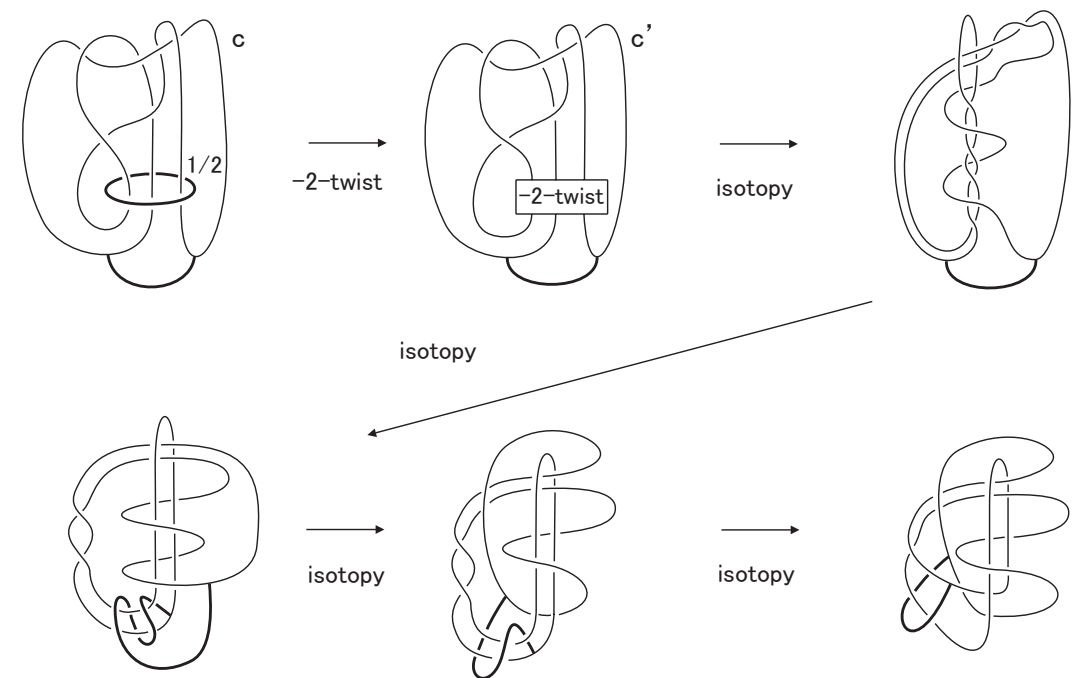

Figure 6. Continued from Figure 5 , 


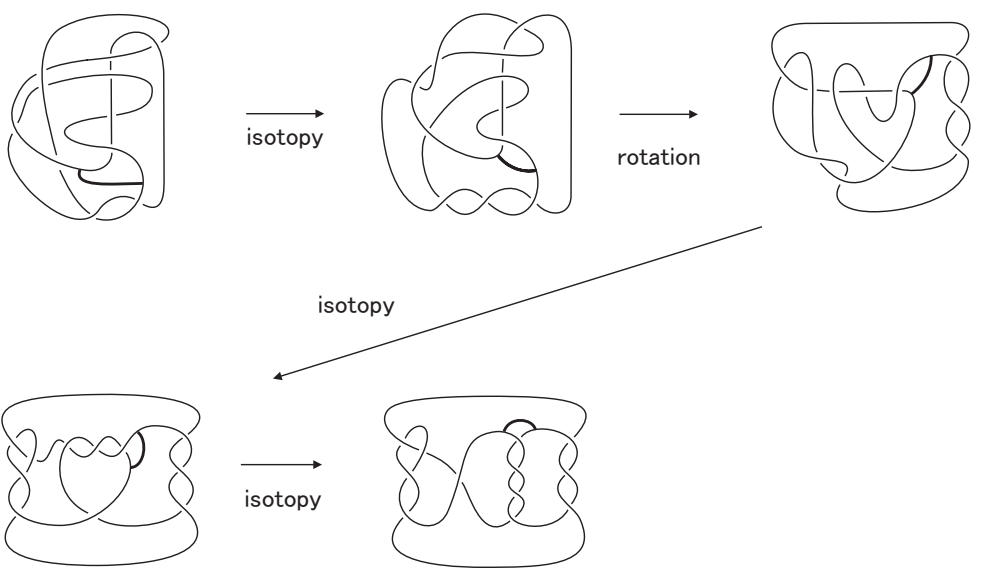

Figure 7. Continued from Figure 6 .

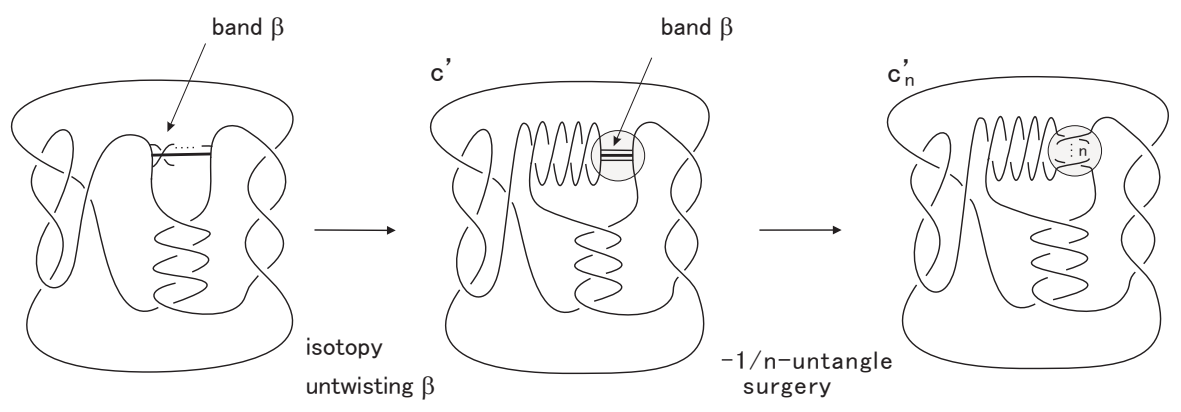

Figure 8. Continued from Figure 7 .

Claim 2.3. $K_{n}$ is a hyperbolic knot.

Proof. The knot $K$ bounds an obvious Seifert surface $S$ of genus one. Since $t_{1}$ can be isotoped off $S$, after doing $n$-twisting along $t_{1}, S$ becomes a Seifert surface for $K_{n}$. By Claim 2.2. $K_{n}$ is a nontrivial knot and thus $g\left(K_{n}\right)$, the genus of $K_{n}$, is equal to one.

Assume for a contradiction that $K_{n}$ is a satellite knot. Then since $\left(K_{n} ; 1\right)$ is atoroidal, $K_{n}$ has a companion solid torus $V$ whose core is a simple knot $\widehat{K_{n}}$ such that $K_{n}$ is a 0 or 1-bridge braid in $V$ ([16, Proposition 2.2(1)]). From Schubert's formula [23] ([6. Proposition 2.10]) we have $g\left(K_{n}\right) \geq w g\left(\widehat{K_{n}}\right)$, where $w$ denotes the winding number of $K_{n}$ in $V$. Since $w \geq 2$ and $g\left(\widehat{\widehat{K}_{n}}\right) \geq 1$, we have $g\left(K_{n}\right) \geq 2$, a contradiction. If $K_{n}$ is a torus knot, then since the genus is one, $K_{n}$ is a $( \pm 2,3)$ torus knot $T_{ \pm 2,3}$. However, $\left(T_{2,3} ; 1\right)$ (resp. $\left(T_{-2,3} ; 1\right)$ ) is a Seifert fiber space of type $S^{2}(2,3,5)$ (resp. $\left.S^{2}(2,3,7)\right)$, contradicting Claim 2.2. It follows that $K_{n}$ is a hyperbolic knot.

$\square($ Claim 2.3)

Claim 2.4. $K_{n}$ is not strongly invertible.

Proof. Recall that $K_{n}$ has cyclic period 2 and that $\left(K_{n} ; 1\right)$ is a Seifert fiber space of type $S^{2}(3,5,|15 n+4|)($ Claim $[2.2)$. Since $|15 n+4|>2$ and $|15 n+4| \neq 3,5$, 
if $K_{n}$ is strongly invertible, then by [22, Theorem 1.7(1)], $K_{n}$ is a torus knot or a cable of a torus knot. This contradicts $K_{n}$ being hyperbolic (Claim 2.3). Therefore $K_{n}$ is not strongly invertible.

$\square$ (Claim 2.4)

Claim 2.5. The tunnel number of $K_{n}$ is two.

Proof. Let $H$ be a handlebody in $S^{3}$ which is obtained by thickening the obvious genus one Seifert surface for $K$. Then $F=\partial H$ is a genus 2 Heegaard surface for $S^{3}$ which contains $K$. Since $t_{1}$ is a core of a handlebody $H, H$ remains a handlebody after $-1 / n$-surgery on $t_{1}$. It follows that $K_{n}$ is embedded in a genus 2 Heegaard surface $F$. Then, by [20, Fact on p. 138] the tunnel number of $K_{n}$ is less than or equal to 2 . On the other hand, since a tunnel number one knot is strongly invertible ([21. Lemma 5]), Claim 2.4 implies that the tunnel number of $K_{n}$ is two.

(Claim 2.5)

Example 2. The second example is a variant of Example 1. Let us consider the trivial knot $t_{2}$ of Figure 9, instead of $t_{1}$ of Figure 1.

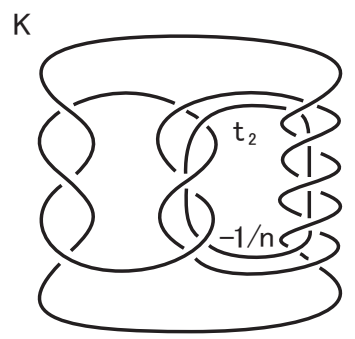

Figure 9.

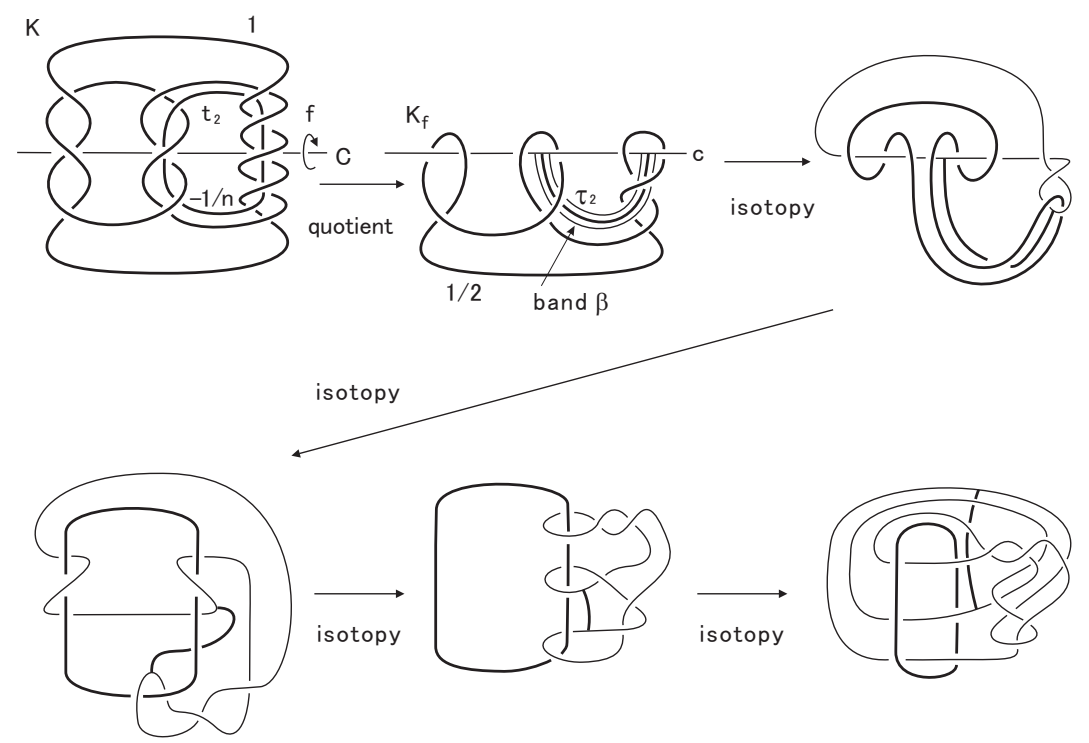

Figure 10. 
Let $K_{n}^{\prime}$ be the knot obtained from $K$ by doing $n$-twisting along $t_{2}$. Then the argument in the proof of Claim 2.2 shows that $\left(K_{n}^{\prime} ; 1\right)$ is a Seifert fiber space of type $S^{2}(3,4,|12 n+5|)$; see Figures 10 13. The arguments in the proofs of Claims 2.1, 2.3, 2.4 and 2.5 show that the $K_{n}^{\prime}$ also enjoy the same properties as in Example 1, and that the Seifert fibered surgeries do not come from the primitive/Seifert-fibered construction.

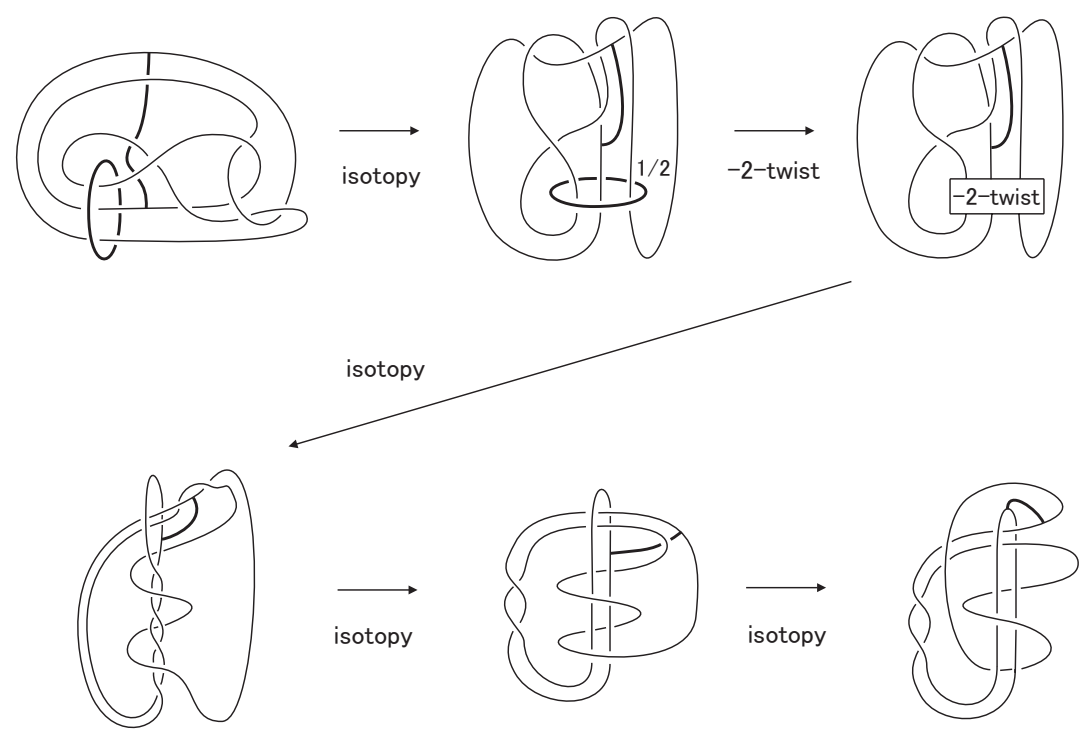

Figure 11. Continued from Figure 10,

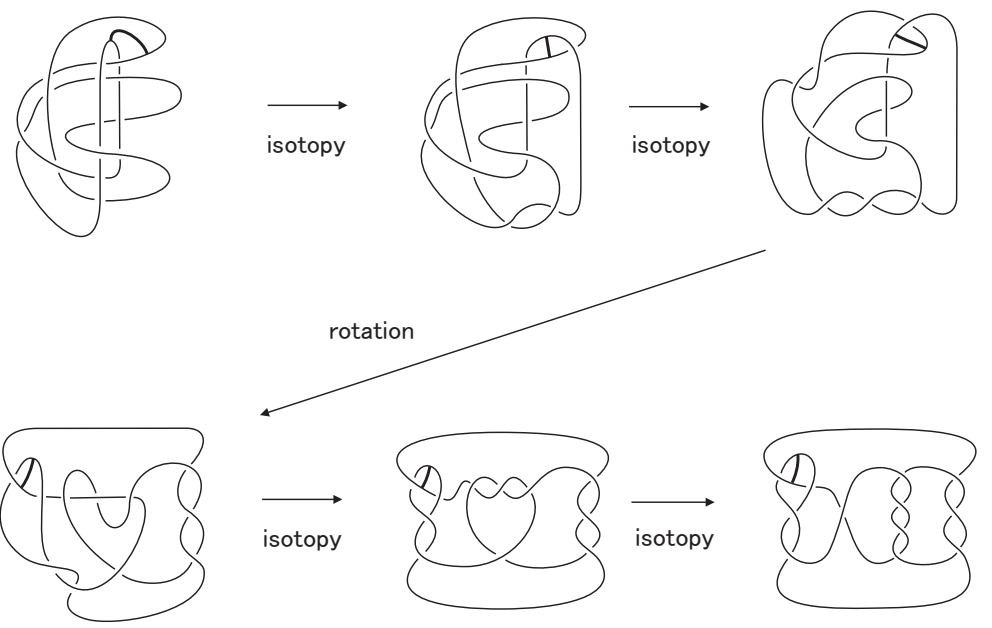

Figure 12. Continued from Figure 11. 


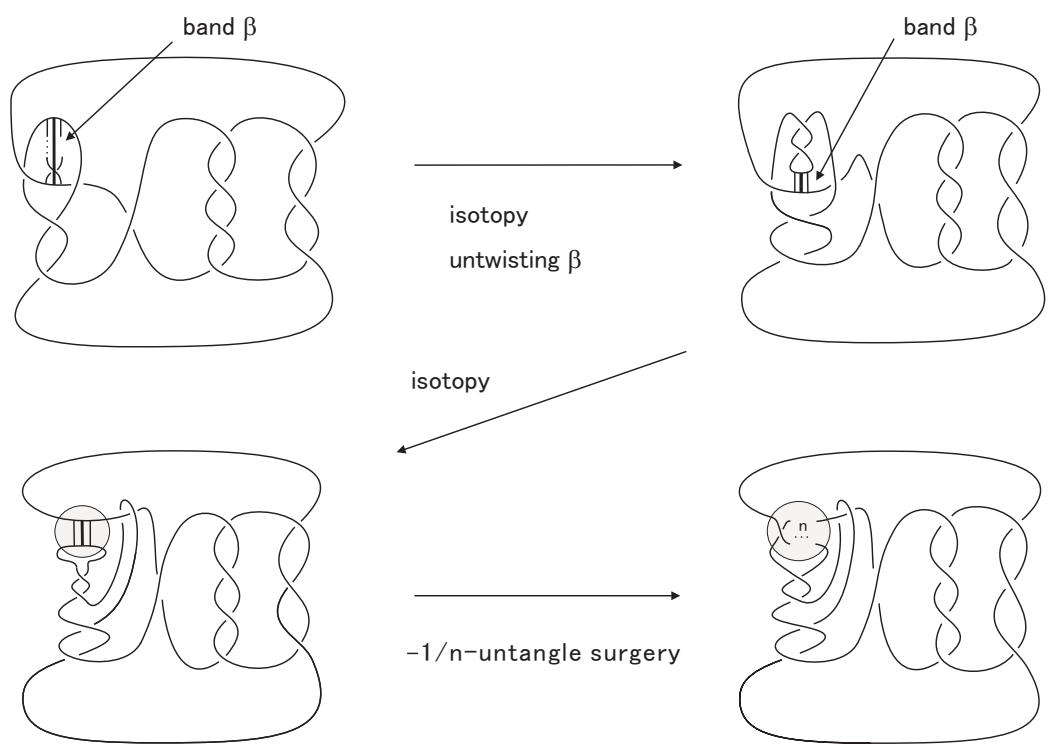

Figure 13. Continued from Figure 12,

\section{REMARKS AND QUESTIONS}

In 17] it has been conjectured that if $(K ; r)$ is a Seifert fiber space, then it admits a Seifert fibration such that one of its fibers is unknotted in (the original) $S^{3}$. For our knots $K_{n}$ (resp. $K_{n}^{\prime}$ ), the trivial knot $t_{1}^{*}$ which is the dual of $t_{1}$ (i.e., the core knot of $-1 / n$-filling along $t_{1}$ ) (resp. $t_{2}^{*}$ which is the dual of $t_{2}$ ) becomes an exceptional fiber of index $|15 n+4|$ in $\left(K_{n} ; 1\right)$ (resp. an exceptional fiber of index $|12 n+5|$ in $\left.\left(K_{n}^{\prime} ; 1\right)\right)$. Thus the Dehn surgeries described in Examples 1 and 2 satisfy the conjecture. (Song's example mentioned in the Introduction also satisfies the conjecture.)

We also mention a geometric aspect of Seifert-fibered surgeries on hyperbolic knots. It was observed in [17, Section 7] that short closed geodesics in hyperbolic knot complements are often unknotted in $S^{3}$ and become Seifert fibers in the resulting Seifert fiber spaces after Dehn surgery. An experiment via Weeks' computer program SnapPea 24] suggests the table below, where $K$ is the $(-3,3,5)$-pretzel knot, and $t_{1}, t_{2}$ are trivial knots described in Figures 1 and 9 . Recall that $(K ; 1)$ is a Seifert fiber space of type $S^{2}(3,4,5)$.

\begin{tabular}{|c|c|c|c|}
\hline & $S^{3}-K$ & $S^{3}$ & $(K ; 1)$ \\
\hline$t_{1}$ & third shortest geodesic & unknot & fiber of index 4 \\
$t_{2}$ & shortest geodesic & unknot & fiber of index 5 \\
\hline
\end{tabular}

The second shortest geodesic is unknotted in $S^{3}$, but it does not become a fiber in $(K ; 1)$. In fact, it is hyperbolic in $(K ; 1)$.

We conclude this paper with some questions. Although the knots given in Examples 1 and 2 cannot be primitive/Seifert-fibered for any genus two Heegaard 
surface, they are still embedded in a genus two Heegaard surface for $S^{3}$. We would like to ask:

Question 3.1. If $(K ; r)$ is a Seifert fiber space, then is $K$ embedded in a genus two Heegaard surface for $S^{3}$ ?

In particular,

Question 3.2. If $(K ; r)$ is a Seifert fiber space, then is the tunnel number of $K$ at most 2?

\section{ACKNOWLEDGEMENTS}

The first author wishes to thank Steven Boyer and Jinha Jun for helpful conversations. We would like to thank the referee for careful reading and useful comments.

\section{REFERENCES}

[1] J. Berge; Some knots with surgeries yielding lens spaces, unpublished manuscript.

[2] S. A. Bleiler; Knots prime on many strings, Trans. Amer. Math. Soc. 282 (1984), 385-401. MR0728719 (85h:57003)

[3] S. A. Bleiler; Prime tangles and composite knots, Lect. Notes in Math., vol. 1144, SpringerVerlag, 1985, pp. 1-13. MR0823278 (87e:57006)

[4] S. Bleiler and C. Hodgson; Spherical space forms and Dehn filling, Topology 35 (1996), 809-833. MR1396779 (97f:57007)

[5] S. Boyer and X. Zhang; Finite surgery on knots, J. Amer. Math. Soc. 9 (1996), 1005-1050. MR1333293 (97h:57013)

[6] G. Burde and H. Zieschang; Knots, de Gruyter Studies in Mathematics 5, 1985. MR0808776 (87b:57004)

[7] J. Dean; Hyperbolic knots with small Seifert-fibered Dehn surgeries, Ph.D. thesis, University of Texas at Austin, 1996.

[8] J. Dean; Small Seifert-fibered Dehn surgery on hyperbolic knots, Algebraic and Geometric Topology 3 (2003), 435-472. MR.1997325 (2004m:57009)

[9] M. Eudave-Muñoz; Non-hyperbolic manifolds obtained by Dehn surgery on a hyperbolic knot, In: Studies in Advanced Mathematics vol. 2, part 1, (ed. W. Kazez), 1997, Amer. Math. Soc. and International Press, pp. 35-61. MR.1470720 (98i:57007)

[10] M. Eudave-Muñoz; On hyperbolic knots with Seifert fibered Dehn surgeries, Topology Appl. 121 (2002), 119-141. MR:1903687 (2003c:57005)

[11] D. Gabai; Foliations and the topology of 3-manifolds III, J. Diff. Geom. 26 (1987), 479-536. MR0910018 (89a:57014b)

[12] F. González-Acuña and H. Short; Knot surgery and primeness, Math. Proc. Camb. Phil. Soc. 99 (1986), 89-102. MR0809502 (87c:57003)

[13] C. McA. Gordon; Dehn Filling; a survey, Knot theory (Warsaw, 1995), 129-144, Banach Center, Publ. 42, Polish Acad. Sci., Warsaw, 1998. MR.1634453 (99e:57028)

[14] C. McA. Gordon and J. Luecke; Knots are determined by their complements, J. Amer. Math. Soc. 2 (1989), 371-415. MR0965210 (90a:57006a)

[15] T. Mattman; The Culler-Shalen seminorms of pretzel knots, Ph.D. thesis, McGill University, Montréal, 2000.

[16] K. Miyazaki and K. Motegi; Seifert fibered manifolds and Dehn surgery II, Math. Ann. 311 (1998), 647-664. MR.1637964 (99g:57010)

[17] K. Miyazaki and K. Motegi; Seifert fibered manifolds and Dehn surgery III, Comm. Anal. Geom. 7 (1999), 551-582. MR1698388 (2000d:57030)

[18] K. Miyazaki and K. Motegi; On primitive/Seifert-fibered constructions, Math. Proc. Camb. Phil. Soc. 138 (2005), 421-435. MR2138571

[19] J. M. Montesinos; Surgery on links and double branched coverings of $S^{3}$, Ann. Math. Studies 84 (1975), 227-260. MR0380802 (52:1699)

[20] K. Morimoto; On the additivity of h-genus of knots, Osaka J. Math. 31 (1994), 137-145. MR:1262793(95d:57006) 
[21] K. Morimoto; There are knots whose tunnel numbers go down under connected sum, Proc. Amer. Math. Soc. 123 (1995), 3527-3532. MR1317043 (96a:57022)

[22] K. Motegi; Dehn surgeries, group actions and Seifert fiber spaces, Comm. Anal. Geom. 11 (2003), 343-389. MR2014880 (2004m:57015)

[23] H. Schubert; Knoten und Vollringe, Acta Math. 90 (1953), 131-286. MR0072482 (17:291d)

[24] J. Weeks; SnapPea: a computer program for creating and studying hyperbolic 3-manifolds, freely available from http://thames.northnet.org/weeks/index/SnapPea.html.

[25] H. Zieschang; On simple systems of paths on complete pretzels, Amer. Math. Soc. Transl. 92, $127-137$.

Department of Mathematics and Statistics, California State University-Chico, Chico, California 95929-0525

E-mail address: TMattman@CSUChico.edu

Faculty of Engineering, TOKyo Denki University, Tokyo 101-8457, Japan

E-mail address: miyazaki@cck.dendai.ac.jp

Department of Mathematics, Nihon University, Tokyo 156-8550, Japan

E-mail address: motegi@math.chs.nihon-u.ac.jp 